ARTICLE

Received 23 Apr 2013 | Accepted 10 Oct 2013 | Published 11 Nov $2013 \quad$ DOl: 10.1038/ncomms3754

\title{
A Nodal-independent and tissue-intrinsic mechanism controls heart-looping chirality
}

Emily S. Noël ${ }^{1}$, Manon Verhoeven ${ }^{1}$, Anne Karine Lagendijk ${ }^{1}{ }^{\dagger}$, Federico Tessadori ${ }^{1}$, Kelly Smith ${ }^{1}{ }^{\dagger}$, Suma Choorapoikayil ${ }^{1, \dagger}$, Jeroen den Hertog $^{1} \&$ Jeroen Bakkers ${ }^{1,2}$

Breaking left-right symmetry in bilateria is a major event during embryo development that is required for asymmetric organ position, directional organ looping and lateralized organ function in the adult. Asymmetric expression of Nodal-related genes is hypothesized to be the driving force behind regulation of organ laterality. Here we identify a Nodal-independent mechanism that drives asymmetric heart looping in zebrafish embryos. In a unique mutant defective for the Nodal-related southpaw gene, preferential dextral looping in the heart is maintained, whereas gut and brain asymmetries are randomized. As genetic and pharmacological inhibition of Nodal signalling does not abolish heart asymmetry, a yet undiscovered mechanism controls heart chirality. This mechanism is tissue intrinsic, as explanted hearts maintain ex vivo retain chiral looping behaviour and require actin polymerization and myosin II activity. We find that Nodal signalling regulates actin gene expression, supporting a model in which Nodal signalling amplifies this tissue-intrinsic mechanism of heart looping.

\footnotetext{
${ }^{1}$ Hubrecht Institute-KNAW and University Medical Center Utrecht, 3584CT Utrecht, The Netherlands. ${ }^{2}$ Interuniversity Cardiology Institute of the Netherlands, 3511GC Utrecht, The Netherlands. † Present addresses: Institute for Molecular Bioscience, University of Queensland, St Lucia, Queensland 4072, Australia (A.K.L. and K.S.); Université Montpellier II, 34095 Montpellier, Cedex 5, France (S.C.). Correspondence and requests for materials should be addressed to J.B. (email: j.bakkers@hubrecht.eu).
} 
A lthough vertebrates appear externally symmetrical, asymmetries exist within the body cavity. To date, many studies have sought to understand the origins of left-right asymmetry determination in a variety of organisms. Embryonic asymmetric expression of Nodal-related genes is evolutionarily conserved and has been described in a large number of organisms, from snails and sea urchins to chickens and mice ${ }^{1-5}$. Knockdown experiments performed in a range of vertebrates have led to a model, wherein this conserved asymmetric Nodal expression is hypothesized to be the driving force behind regulation of organ laterality ${ }^{1,2,6-8}$. However, these studies have been hampered because of an early requirement for Nodal signalling in primitive streak formation and mesoderm specification ${ }^{9}$.

In zebrafish, three homologues of Nodal have been reportedcyclops (cyc), squint (sqt) and southpaw (spaw) ${ }^{10,11}$. Although cyc and sqt are expressed during gastrulation and are required for mesendoderm specification, spaw is expressed around the Kupffers vesicle and in the left lateral plate mesoderm (LPM) during somitogenesis stages ${ }^{10,11}$. Morpholino oligonucleotidemediated knockdown of spaw results in loss of asymmetric gene expression and disruption of organ laterality, demonstrating a role for spaw in the regulation of organ asymmetry ${ }^{11}$.

Here we present the first described spaw mutant. We show that although intestinal and brain laterality is randomized in spaw mutants, the heart exhibits a previously undescribed preferential looping direction, with the majority of hearts looping in the correct orientation. Genetic and pharmacological inhibition of Nodal signalling demonstrates that this directional bias is independent of Nodal activity. We further show through compound mutant analyses that this directional bias is partly dependent on fluid flow in the Kupffer's vesicle (KV). Using an ex-vivo heart culture system, we demonstrate that dextral heart looping is a Nodal-independent and tissue-intrinsic process, requiring actomyosin activity. Finally, we show that Nodal signalling regulates actin gene expression, and suggest that asymmetric Nodal signalling may enhance a cytoskeleton-based tissue-intrinsic mechanism of heart looping.

\section{Results}

The straightforward mutation affects organ laterality. The straightforward (sfw) mutant was identified in a forward genetic

\section{Figure 1 | The sfw mutant displays heterotaxia due to loss of Spaw}

activity. (a) Schematic depicting cardiac asymmetries observed during zebrafish development. At $28 \mathrm{hpf}$, the heart is a linear tube positioned under the left eye, which normally undergoes dextral looping by $48 \mathrm{hpf}$. (b) Analysis of heart position at $28 \mathrm{hpf}$ by in situ analysis of myl7 expression, dorsal views, anterior to top. (c) Bright-field images of wild-type (wt) and sfw mutant embryos at $48 \mathrm{hpf}$. (d) Quantification of heart-looping direction at $48 \mathrm{hpf}$ in wt and sfw mutant embryos. Heart is highlighted by myl7 expression. (e) Quantification of digestive system laterality at $48 \mathrm{hpf}$ in wt embryos and sfw mutants, using foxA3 in situ hybridization to visualize the digestive tract. (f) Quantification of habenulae laterality in wt and sfw mutant embryos at $4 \mathrm{dpf}$ by lov in situ hybridization. (d-f) Error bars indicate s.e.m. Significance determined by Student's $t$-test indicated by asterisks: ${ }^{\star} P<0.05,{ }^{\star \star} P<0.01,{ }^{\star \star \star} P<0.005$. (d,e) On the basis of 25 biological replicates (total embryo number $=796$ ), (f) based on 3 biological replicates (total embryo number 126). L, liver; P, pancreas; asterisks denote position of lov expression in the brain. (g) The sfw mutation was mapped to linkage group 5. x/96 denotes number of recombinants in 96 meioses. Genomic position is noted in Mb. (h) Amino acid alignment of Nodalrelated genes in zebrafish, Xenopus, mouse and human. The mutated cysteine residue is highlighted by the red box and is conserved in all Nodals. Scale bars, $100 \mu \mathrm{M}(\mathbf{b}, \mathbf{c})$ screen for mutants with organ laterality defects ${ }^{12}$. Whereas the heart in wild-type embryos is positioned asymmetrically under the left eye at $28 \mathrm{~h}$ post fertilization (hpf), sfw mutants exhibit a midline heart at $28 \mathrm{hpf}$ (Fig. 1a,b) accompanied by no other morphological defects (Fig. 1c). Surprisingly, the sfw mutant phenotype displays an uncoupling of cardiac positioning and cardiac looping events; although the initial cardiac tube position (at $28 \mathrm{hpf}$ ) is strongly affected in the $s f w$ mutant embryos, cardiac looping (at $48 \mathrm{hpf}$ ) is only mildly affected. Analysis of heart laterality at $48 \mathrm{hpf}$ demonstrated that directional looping occurs in $93 \%$ of the $s f w$ mutant embryos. Interestingly, in $70 \%$ of $s f w$ mutant embryos cardiac looping occurs in the correct dextral

a Dorsal view (28 hpf) Ventral view (48 hpf)

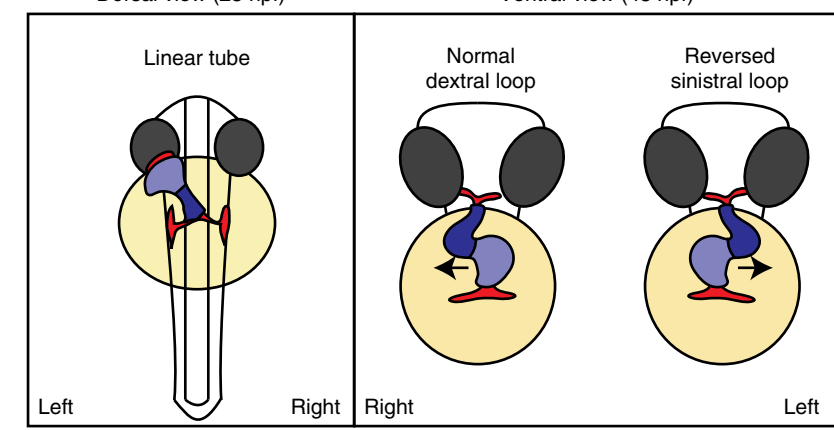

b

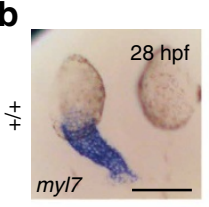

C
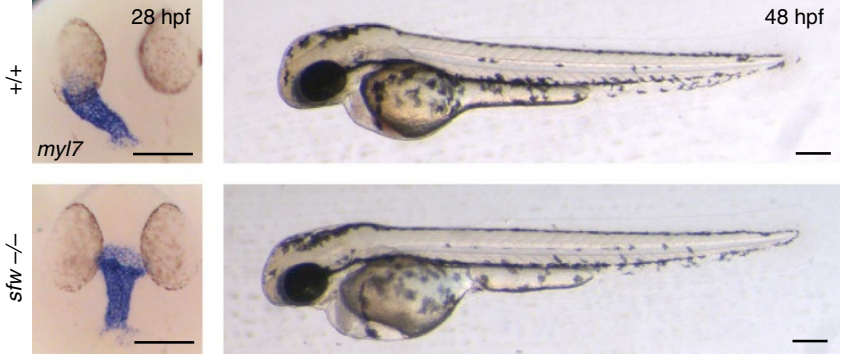

d

Heart 48 hpf
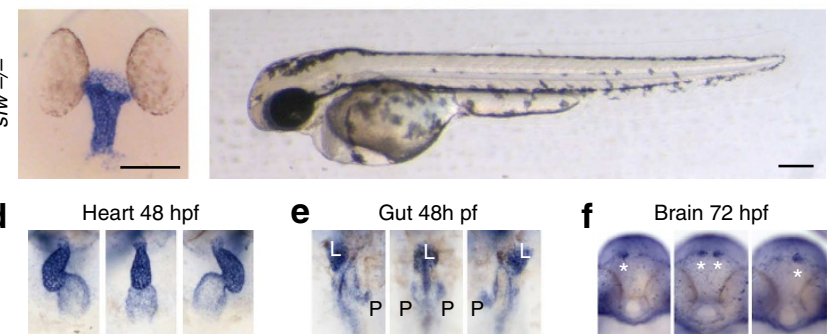

e

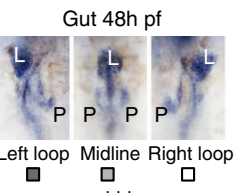

f

d-Loop No loop s-Loop

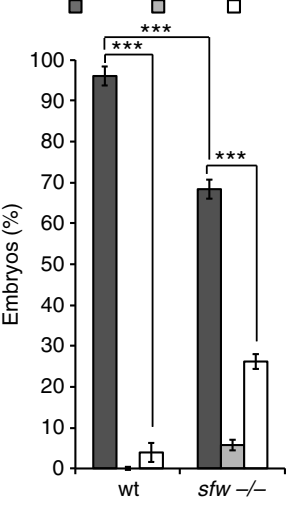

Left
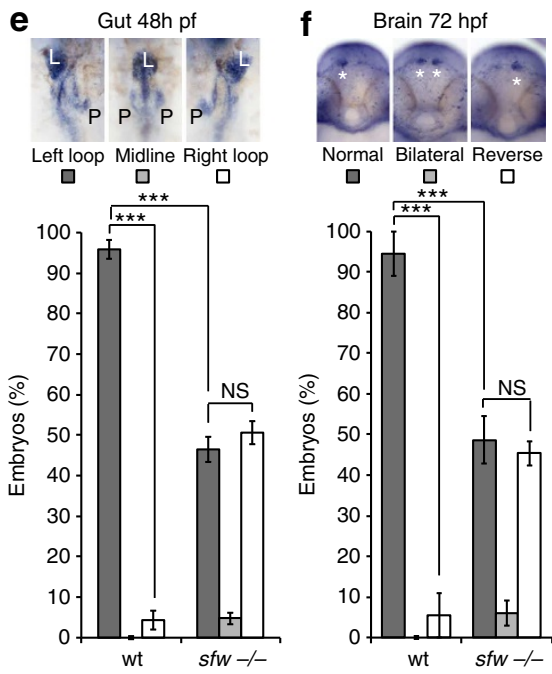

Normal Bilateral Reverse

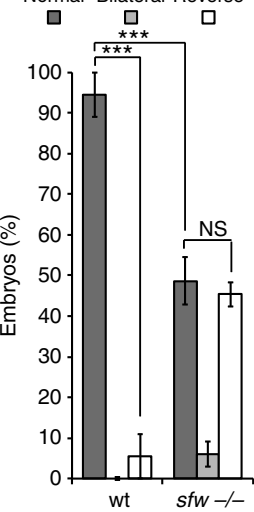

g

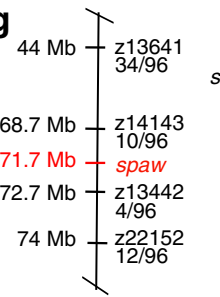

h

southpaw ${ }^{\text {C401F }}$ D.rerio MLYYEGDGVVMRHHE DMI VEEFG GCH southpaw D.rerio MLYYEGDGVVMRHHE DMI VEECGCH squint D.rerio ML YY EN GK MVMR HHE GMVVAE C GCH cyclops D.rerio ML YY EN GEMI LRHHE DMQVEECG CL Xnr1 X. tropicalis MLYY E GDEVVLRHHQE MI VEECGCS Xnr3A X.laevis ML YYENEDF I LRHHEEMI VEECGFK Nodal M.musculus ML YVDNGRVL LEHHK DMI VEE CGCL Nodal H.sapiens MLYVDNGRVL LDHHK DMI VEECGCL 
direction (d-loop; Fig. 1d, $n=796$ ). Expression analysis of marker genes for the atrioventricular canal, working myocardium, endocardium and valves revealed no other cardiac defects in sfw mutant embryos (Supplementary Fig. S1). Interestingly, analysis of endodermal organ positioning in $s f w$ mutants revealed a randomization of digestive system laterality (Fig. 1e, $n=796$ ). In addition, we analysed the left-right asymmetry in the brain by assessing the expression of leftover (lov), which is expressed in the left habenula of wild-type embryos at 3 days post fertilization $(\mathrm{dpf})^{13}$, and observed a randomization of lov expression in $s f w$ mutants (Fig. 1f; $n=126$ ).

To determine whether aberrant left-right patterning was responsible for the observed organ laterality defects, we analysed the expression of known laterality genes. In wild-type embryos, the Nodal-related gene, spaw, is robustly expressed around the $\mathrm{KV}$ (the equivalent organ to the node for the zebrafish) and in the left LPM (Fig. 2e,f) ${ }^{11}$. We found that although spaw expression was initiated around the KV and in the posterior LPM in sfw mutant embryos at 10 somites $(64 \%, n=149$, Fig. $2 \mathrm{~g})$, by $18 \mathrm{hpf}$ (18 somites) spaw expression had not propagated anteriorly in the left LPM of $s f w$ mutants (100\%, $n=95$; Fig. 2h). Similarly, lefty1, a Nodal antagonist expressed in the midline of wild-type embryos (Fig. 2a,b), was induced but not maintained nor propagated anteriorly in the midline of $s f w$ mutants (Fig. $2 c, d$ ). Consistent with the loss of spaw expression in the left anterior LPM of $s f w$ mutant embryos, we observed a loss of asymmetric gene expression in the organ anlage, such as lefty 2 in the heart, the Nodal-related gene cyclops in the heart and brain, and the Nodalregulated gene, pitx2, in the endodermal organ region, (Fig. 2i-p). From this analysis, we concluded that the mutation responsible for the $s f w$ phenotype is acting either upstream or at the same level as Nodal signalling. Despite the severe organ laterality defects, which were most often discordant (heterotaxia, Supplementary Table S1), sfw mutant embryos were adult viable. Maternal and zygotic sfw mutant embryos derived from in-crosses of $s f w$ mutant adults displayed no additional morphological phenotypes (Supplementary Fig. S2).

To determine the position of the mutation underlying the $s f w$ mutant phenotype, we undertook a positional cloning approach. Bulked segregant analysis placed the mutation onto linkage group 5 , between markers z13641 and z22152. Fine mapping with simple sequence length polymorphism (SSLP) markers narrowed the region down to a $4-\mathrm{Mb}$ region, including the spaw gene locus (Fig. 1g). Sequencing of the entire spaw coding sequence in sfw mutants revealed a single base-pair change that always cosegregated with the $s f w$ mutant phenotype (Supplementary Fig. S3). The identified variant causes a substitution of a conserved cysteine with a phenylalanine at residue 401 of the carboxy terminus of the mature transforming growth factor $\beta$ domain (Fig. 1h). Although knockdown of Spaw using antisense morpholino oligonucleotides has been shown to result in disrupted organ laterality ${ }^{11}$, we wished to confirm that the uncoupling of cardiac tube position and cardiac looping observed in sfw mutant embryos was due to loss of Spaw function. Analysis of cardiac tube position and cardiac looping in embryos injected with spaw morpholino revealed that although $>90 \%$ of cardiac tubes remained midline, normal dextral heart looping was observed in a majority of spaw morpholino-injected embryos as was observed for $s f w$ mutant embryos (Fig. 1 and Supplementary Fig. S3). To confirm that the Nodal pathway was disrupted in sfw mutants, we implanted beads soaked in recombinant Nodal protein into the left side of the embryos at the cardiac disc stage before the onset of cardiac tube formation. Implantation of a Nodal bead into sfw mutant embryo completely rescued asymmetric heart tube position when compared with nonimplanted controls, demonstrating that the Nodal pathway is
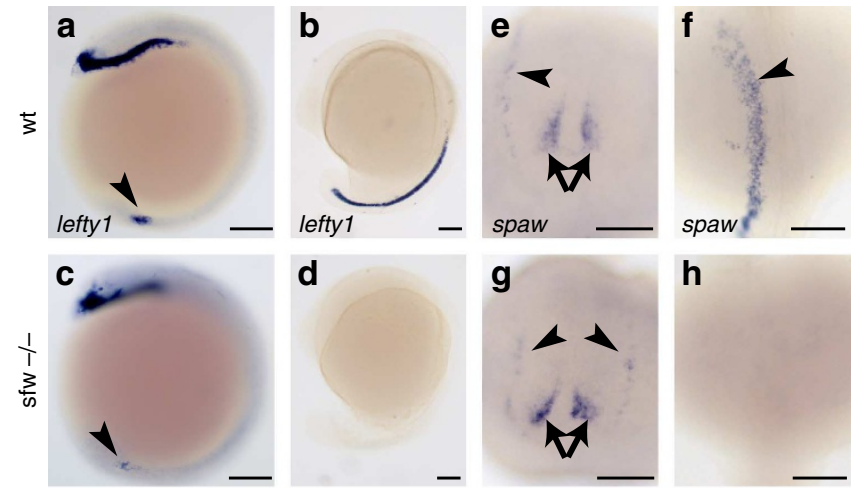

d
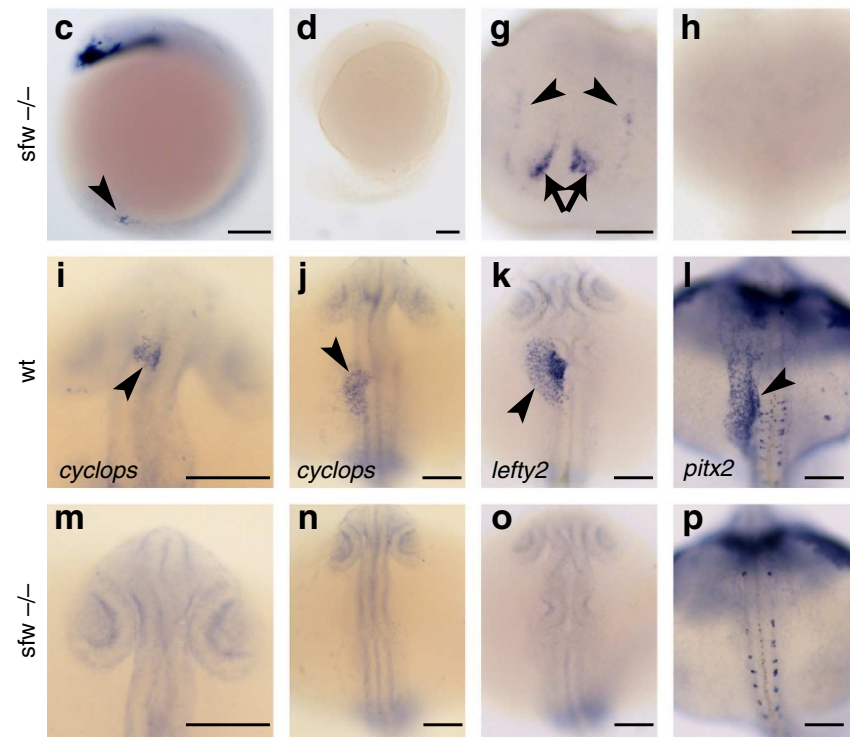

Figure 2 | Expression of laterality genes is lost in sfw mutants. (a-d) In situ hybridization analysis of leftyl expression in wild-type $(\mathbf{a}, \mathbf{b})$ and $s f w$ mutants (c,d). lefty 1 is initiated in the caudal notochord in wild-type embryos at $12 \mathrm{hpf}$ ( 5 somites; $\mathbf{a}$, arrowhead) and its expression expands anteriorly in the midline over the course of somitogenesis (b, $16 \mathrm{hpf}, 15$ somites). In sfw mutants, lefty1 expression is initiated in the midline at $12 \mathrm{hpf}$ (5 somites; c, arrowhead); however, it is not expressed in the midline at $16 \mathrm{hpf}$ (d). (e,f) spaw expression starts around the KV (arrows) and is initiated in the LPM (arrowhead) of wild-type embryos at (14 hpf, 10 somites) (e) and propagates anteriorly during somitogenesis (16 hpf, 15 somites; $\mathbf{f}$, arrowhead). In sfw mutants, spaw expression is initiated around the KV (arrows) and in the posterior LPM (arrowheads) at $14 \mathrm{hpf}$ (g); however, spaw expression does not spread to anterior parts of the LPM later during somitogenesis (h). (i,j) cyclops is expressed in the left diencephalon (i) and left heart field (j) in wild-type embryos at $21 \mathrm{hpf}$ (23 somites), but is absent from both domains in sfw mutant embryos $(\mathbf{m}, \mathbf{n})$. (k, $\mathbf{l})$ Wild-type embryos express lefty2 in the left heart field (k) and pitx2 in the left LPM (I) at $22 \mathrm{hpf}$ ( 25 somites); however, expression of both lefty 2 in the heart and pitx 2 in the left LPM is absent in sfw mutant embryos (o,p). Scale bars, $100 \mu \mathrm{M}$.

indeed disturbed in sfw mutants (Supplementary Fig. S3). To address whether the identified variant affected Spaw function, we injected wild-type spaw and sfw-spaw RNA into embryos and scored for dorsalization due to ectopic Nodal activity ${ }^{14,15}$. Overexpression of wild-type spaw and sfw-spaw RNA demonstrated that the C401F variation results in a complete loss of function of Spaw activity (Supplementary Fig. S3).

Preferred dextral looping is independent of Nodal signalling. As preferred dextral heart looping was maintained in sfw mutants, we wanted to exclude that this resulted from Nodal activity from other Nodal-related proteins such as Cyclops and Squint ${ }^{10}$. We therefore blocked Nodal signalling in $s f w$ mutants using the chemical SB431542 that inhibits the Nodal receptor, Alk7 (ref. 16), or by temporally controlled overexpression of the Nodal antagonist, lefty1 (Supplementary Fig. S4). To confirm that 
application of SB431542 or overexpression of lefty1-blocked Nodal signalling, we used both techniques at blastula stages and assessed mesoderm formation at $24 \mathrm{hpf}$, or applied these techniques at the onset of somitogenesis and assessed spaw expression in the LPM at 20 somites. We found that employing both techniques at blastula stages efficiently disrupted formation of mesodermal structures, and that application at the onset of somitogenesis completely abrogated spaw expression, confirming that both techniques effectively block Nodal signalling (Supplementary Fig. S4). We therefore applied SB431542 to wild-type and $s f w$ mutant embryos at $10 \mathrm{hpf}$, or overexpressed leftyl in wild-type and $s f w$ mutant embryos at either $10 \mathrm{hpf}$ or $13 \mathrm{hpf}$, and assessed cardiac-looping direction at $50 \mathrm{hpf}$. Under all conditions, we still observed preferred dextral heart looping (Fig. 3a,b and Supplementary Table S2). Furthermore, reverse transcription-PCR (RT-PCR) analysis of expression levels of the Nodal homologues $c y c$ and sqt at stages relevant to heart lateralization (13-28 hpf) in wild-type and $s f w$ mutant embryos demonstrated an absence of these genes in sfw mutants (Supplementary Fig. S4), together supporting a model in which d-looping in the absence of Nodal signalling is controlled by an alternative mechanism.

Mutant embryos with disrupted cilia-mediated fluid flow in the $\mathrm{KV}$ display a wide variety of laterality defects ${ }^{17-19}$. To determine whether the residual cardiac-specific directional bias in sfw mutants is the result of alternative signals under the control of $\mathrm{KV}$ function, we analysed heart-looping direction in dnaaf 1 mutants with immotile cilia ${ }^{18,19}$. Surprisingly, dnaaf 1 mutants or sfw/dnaaf 1 double-mutant embryos still exhibited a significant preferred dextral heart loop (Fig. $3 c$ and Supplementary
Table S2), indicating the existence of a mechanism driving directional heart looping independently of both cilia-induced fluid flow and Nodal signalling.

Cardiac disc rotation is dispensable for dextral looping. During heart tube formation, the cardiac disc undergoes an asymmetric tissue rearrangement that includes rotation of the cardiac disc and local invagination at the right side. This reorganization results in the left side of the disc, ultimately forming the dorsal face of the heart tube ${ }^{19-21}$. It has been suggested that such rotational events may provide directed torsion in the heart tube, which in turn may drive directional looping ${ }^{20,22-25}$. Previous studies on heart tube formation using spaw knockdown experiments have suggested that in the absence of Nodal, both the direction of cardiac disc rotation and the site of myocardial involution are randomized, resulting in either a leftward or rightward positioning of the cardiac tube $11,19,21,26$. Our observation that the linear heart in $s f w$ mutants is predominantly positioned at the midline and rarely under the left or right eye suggests that the reported randomization in heart position was due to an incomplete spaw knockdown.

To determine whether cardiac disc rotation occurs in $s f w$ mutants and whether any degree of rotation is significantly linked to subsequent laterality of heart looping, we imaged heart tube formation in wild-type embryos or sfw mutant embryos between $20 \mathrm{hpf}$ and $24 \mathrm{hpf}$ (Fig. 4a), and subsequently raised the embryos to $55 \mathrm{hpf}$ when handedness of cardiac looping could be determined. We tracked individual myocardial cells during linear heart tube formation (Fig. 4a) and measured their displacement
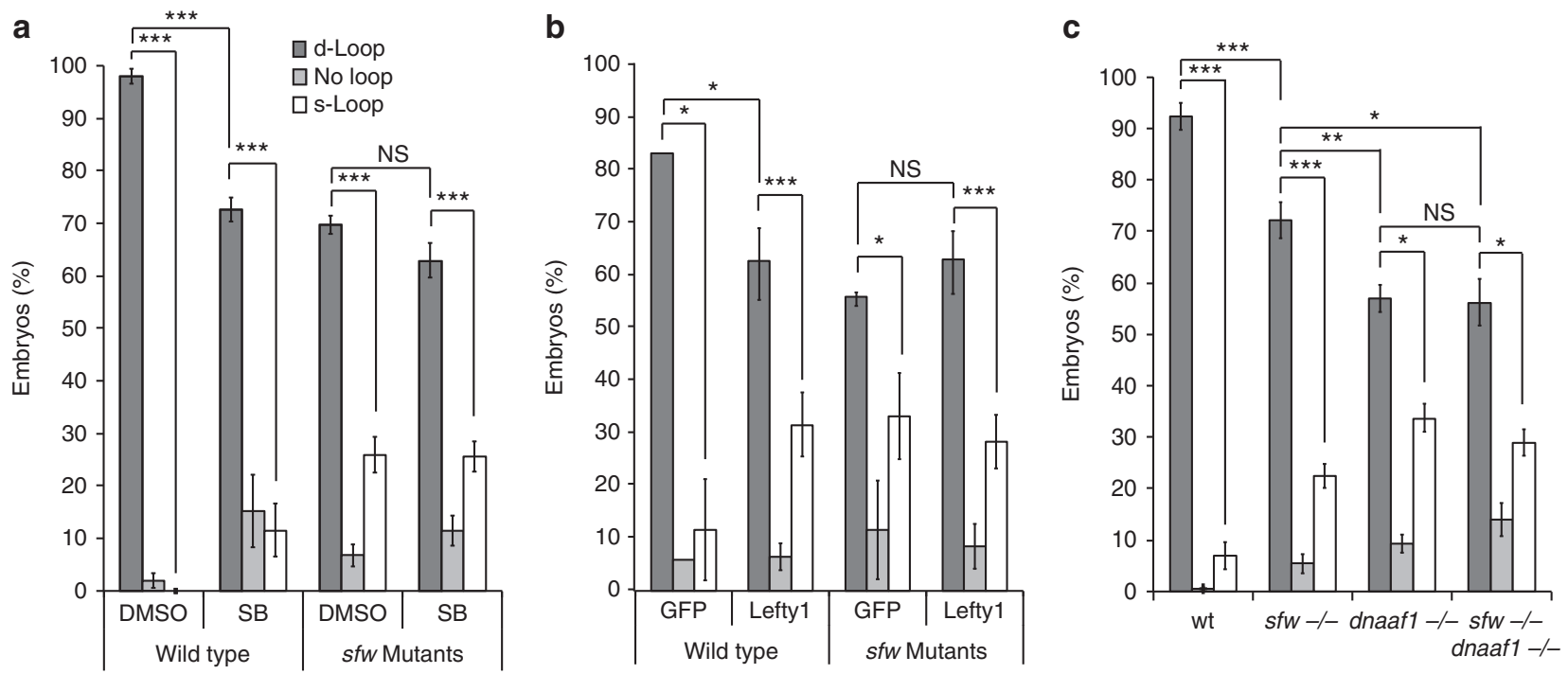

Figure 3 | Dextral looping bias in sfw mutants occurs independently of Nodal activity or KV function. (a) Analysis of looping direction in wild-type (wt) embryos or sfw mutants treated with the Nodal inhibitor SB431542. Wild-type embryos treated with SB431542 show a significant reduction in the number of $d$-looped hearts when compared with dimethyl sulphoxide (DMSO)-treated controls. However, no significant reduction in the number of $d$-looped hearts is observed in sfw mutant embryos treated with SB431542 when compared with DMSO-treated controls; $n=7$ clutches of embryos. (b) Analysis of looping direction in wt embryos and sfw mutants, injected with either a uas:gfp control or injected with uas:Ift1 and heat-shocked at 14 hpf (8-10 somite stage). Wild-type embryos overexpressing uas:Ift1 showed a significant reduction in the number of $d$-looped hearts when compared with uas:gfpinjected controls. No significant reduction in the number of d-looped hearts was observed in sfw mutants injected with uas:Ift 1 when compared with uas:gfpinjected controls. uas:gfp data $n=4$ clutches of embryos, uas:lefty1 data $n=7$. (c) Analysis of looping direction in wt embryos, sfw mutants, dnaaf1 mutants and sfw/dnaaf1 double mutants. sfw mutants show a significant reduction in the number of d-looped hearts when compared with wild-type embryos. Dnaaf1 mutants show a significant reduction in the number of d-looped hearts when compared with either sfw mutants or wild-type embryos. sfw/dnaaf1 double-mutant embryos show a significant reduction in the number of d-looped hearts when compared with sfw mutants or wild-type embryos; however, there is no significant reduction when compared with dnaaf1 single mutants. Sfw mutants, dnaaf1 mutants and sfw/dnaaf1 double mutants all have significantly more dextral looped hearts than sinistral or non-looped hearts; $n=10$ clutches of embryos. (a-c) Error bars indicate s.e.m. Significance determined by Student's $t$-test indicated by asterisks: ${ }^{\star} P<0.05,{ }^{\star \star} P<0.01,{ }^{\star \star \star} P<0.005$. 
a
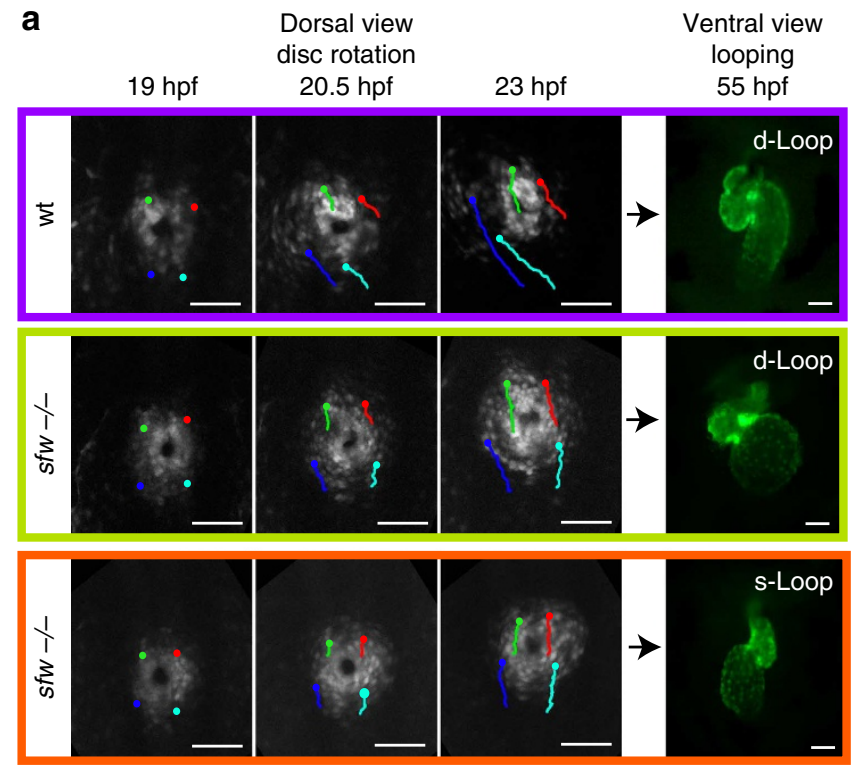

b
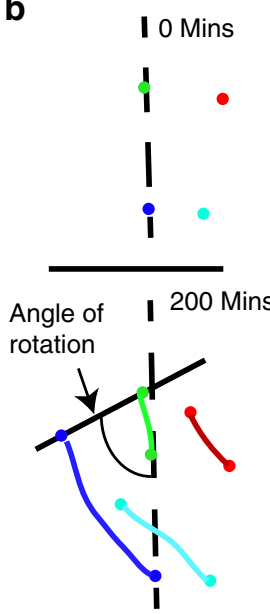

\begin{abstract}
C
\end{abstract}

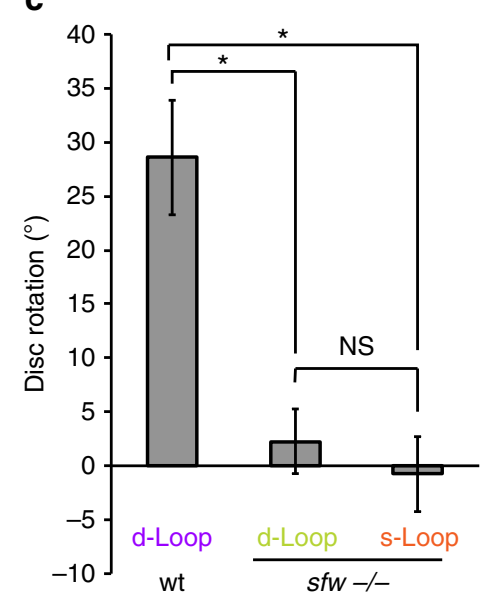

Figure 4 | Chiral heart looping occurs independently from cardiac disc rotation. (a) Still images (dorsal view, anterior up) from time-lapse movies generated from projected confocal stacks using the $\mathrm{Tg}$ (myl7:GFP) transgenic line labelling all cardiomyoscytes. Blue, green, red and turquoise traces indicate movement of individual cells in wild-type or sfw mutants embryos between $19.5 \mathrm{hpf}$ and $23 \mathrm{hpf}$. Ventral view stills indicate direction of heart looping at $55 \mathrm{hpf}$ in time-lapse-imaged embryos. (b) Schematic showing method for analysis of cardiac rotation. (c) Quantification of cardiac disc rotation in wild-type embryos with a d-looped heart, sfw mutants with $\mathrm{d}$-looped heart and sfw mutants with s-looped heart $(n=4$ for each category). Error bars indicate s.e.m. Significance determined by Student's $t$-test indicated by asterisks: ${ }^{\star} P<0.05 ; n=4$ embryos for each category (wild-type, d-loop mutant, s-loop mutant). Scale bars, $50 \mu \mathrm{M}$ (a).

rates and direction (Fig. 4b). We observed that cardiac disc rotation failed to occur in either dextral or sinistral looped hearts of $s f w$ mutant embryos $\left(2.2^{\circ}\right.$ and $-0.8^{\circ}$, respectively, $n=4$ for each; Fig. 4c) and that the overall velocity of cardiomyocytes was significantly slower in $s f w$ mutants (Supplementary Fig. S5). These results demonstrate that cardiac disc rotation is not required for handedness of heart looping.

Heart-intrinsic dextral looping requires actomyosin activity. Isolated linear heart tubes from chick and amphibian embryos maintain their ability to form a dextral loop ex vivo ${ }^{27,28}$. We therefore hypothesized that in the absence of Nodal signalling, preferential dextral cardiac looping is maintained due to an alternative tissue-intrinsic mechanism. To test this hypothesis, we developed an ex-vivo heart culture system by manually dissecting linear heart tubes from zebrafish embryos at $28 \mathrm{hpf}$ and culturing these in supplemented tissue culture medium (Fig. 5a). Indeed, we observed that linear heart tubes of both wild-type embryos and $s f w$ mutant embryos underwent looping after $24 \mathrm{~h}$ in culture (Fig. 5b). Furthermore, we observed that $79 \%$ of explanted wildtype hearts displayed a dextral loop after $24 \mathrm{~h}$ in culture (Supplementary Fig. S6).

In Drosophila and Caenorhabditis elegans, both of which lack Nodal genes in their genome, laterality is controlled by tissue- and cell-intrinsic mechanisms that require specific components of the cytoskeleton $^{29-34}$. Similarly, cultured mammalian cells show phenotype-specific left-right asymmetry that is controlled by the cells cytoskeleton ${ }^{33,34}$. To determine whether the tissue-intrinsic and Nodal-independent mechanism that controls heart looping requires specific components of the cytoskeleton, we treated explants of linear heart tubes with various inhibitors affecting normal cytoskeleton formation. We observed normal heart looping in wild-type and $s f w$ mutant hearts treated with either dimethyl sulphoxide or Nocodazole during the culturing period (Fig. 5b), indicating that microtubule formation is dispensable for normal heart looping. However, explanted hearts incubated with either the myosin II inhibitor, Blebbistatin, or the actin polymerization inhibitor, Cytochalasin B, displayed compromised looping and a failure of constriction at the atrioventricular canal (Fig. 5b), indicating that both the actin cytoskeleton and its interactions with non-muscle myosin II are specifically required for the tissue-intrinsic cardiac remodelling that underlies heart looping. This requirement for myosin II in cardiac looping was also observed in wild-type embryos and is independent from its role in cardiac contractility, as we found that mutant hearts defective in cardiac contractility displayed normal dextral looping (Supplementary Fig. S7).

Nodal signalling regulates actin levels. How can two mechanisms (a Nodal-dependent and a Nodal-independent mechanism) efficiently control handedness of heart looping? The asymmetric Nodal expression in the LPM could amplify the heart-intrinsic mechanism that requires actomyosin activity. Indeed, in chick and mouse embryos with asymmetric Nodal activity, messenger RNA of genes encoding cytoskeleton components or their products localize asymmetrically in the heart tube ${ }^{35,36}$. Several actin-related genes are expressed in the embryonic zebrafish heart ${ }^{37,38}$ and RT-PCR analysis revealed that $\alpha$-actin $1 b$ (acta1b) was downregulated in sfw mutant embryos before heart looping (Fig. 5c). In situ hybridization combined with image analysis revealed that actalb is expressed asymmetrically in the wild-type heart at 23 somites ( $21 \mathrm{hpf})$ before leftward displacement, with elevated expression of actalb on the left side of the cardiac disc compared with the right side (Fig. 5d). This left-sided elevation of actalb was lost in sfw mutants. If the asymmetric Nodal expression in the LPM amplifies a heart-intrinsic mechanism by elevating actin levels, then hearts of sfw mutant embryos lacking asymmetric Nodal activity should be more prone to perturbations of actin polymerization. Corroborating such a model, we observed that treatment of wild-type embryos with a low concentration of Cytochalasin B had no effect on heart looping, whereas the same treatment efficiently perturbed heart looping in $s f w$ mutants (Fig. 5e).

\section{Discussion}

We conclude that chiral heart looping is a tissue-intrinsic process that is controlled by both Nodal-dependent and -independent mechanisms. We suggest the following model (shown in Fig. 5f) 
a

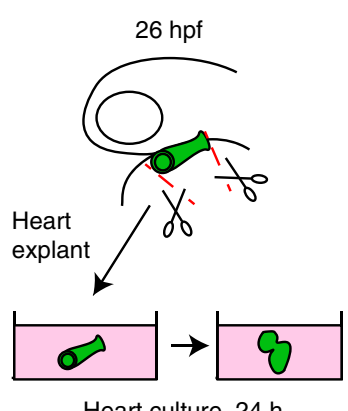

Heart culture, $24 \mathrm{~h}$ b
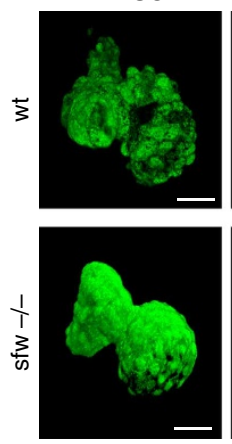

Blebbistatin
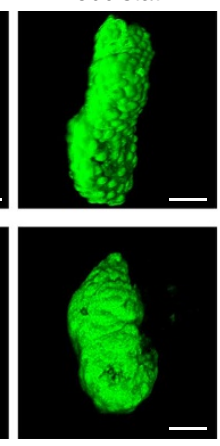

Cytochalasin $\mathrm{B}$
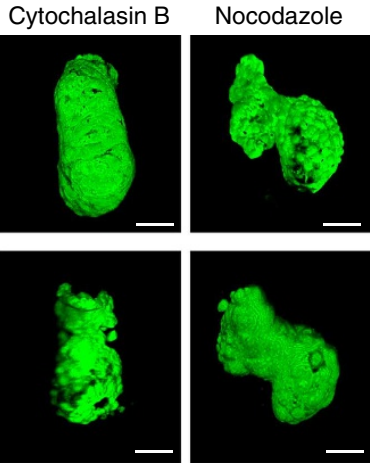

d Left
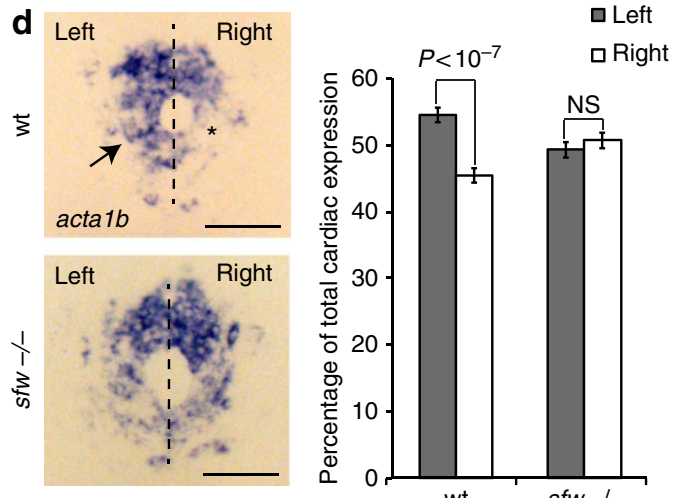

口Right

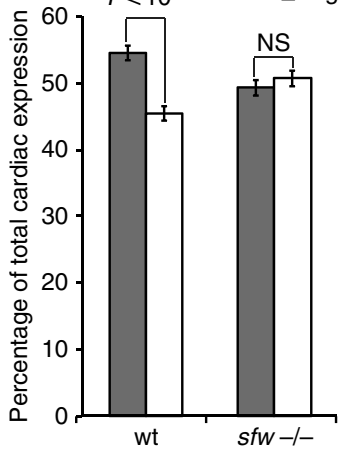

$\mathbf{f}$

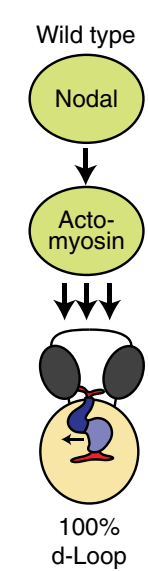

Wild type +

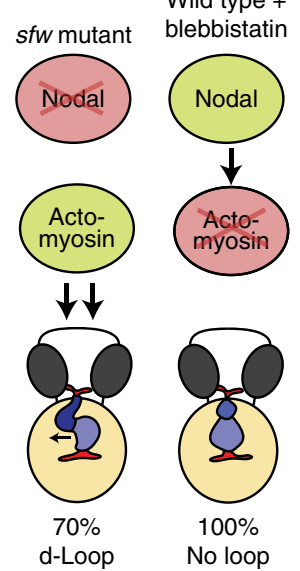

Figure 5 | Heart looping is a tissue-intrinsic process that requires actomyosin activity. (a) Schematic depicting the heart explant system. Linear heart tubes were dissected from $\mathrm{Tg}$ (myl7:GFP)-positive embryos at $26 \mathrm{hpf}$, placed into culture medium and incubated for $24 \mathrm{~h}$ before fixation and imaging. (b) Explanted linear heart tubes of wild-type or sfw mutant embryos were treated with either dimethyl sulphoxide (DMSO), Blebbistatin, Cytochalasin B or Nocodazole during ex vivo culturing for $24 \mathrm{~h}$. (c) Quantitative RT-PCR analysis of relative levels of expression of acta1b, spaw, lefty1 or myod in sfw mutants compared with wild-type embryos at $21 \mathrm{hpf}$ (23 somites). Acta1b, spaw and lefty 1 expression was downregulated in sfw mutant embryos, whereas myoD expression was unaffected. Three biological replicates were used and each biological sample was analysed in triplicate. (d) In situ hybridization analysis of actalb expression in wild-type and sfw mutant embryos at $21 \mathrm{hpf}$. Quantification by image analysis of relative levels of expression between the left and right sides of the cardiac fields revealed that wild-type embryos had elevated actalb expression in the left cardiac field (arrow) compared with the right side (asterisk), whereas sfw mutants had no asymmetric elevation of actalb expression. Thirty-six embryos from two biological replicates were analysed. (e) Heart-looping direction in wild-type embryos or sfw mutants treated with either DMSO or $5 \mu \mathrm{g} \mathrm{ml}{ }^{-1}$ Cytochalasin B between 26 hpf and 55 hpf. Treatment of wild-type embryos with Cytochalasin B had no effect on heart-looping direction, whereas treatment of sfw mutant embryos with Cytochalasin B significantly reduced the number of dextral looped hearts and increased the number of non-looped hearts; $n=9$ clutches of embryos. (c-e) Error bars indicate s.e.m. Significance determined by Student's $t$-test indicated by asterisks: ${ }^{\star \star} P<0.01$. (f) Cartoon illustrating possible regulation of dextral heart looping by Nodal and actomyosin. In a wild-type situation, combined Nodal signalling and actomyosin activity provide a robust mechanism driving dextral heart looping. In the absence of Nodal signalling, actomyosin activity is sufficient to promote preferential dextral heart looping. In the absence of actomyosin activity, Nodal signalling is not sufficient to promote dextral heart looping. Scale bars, $50 \mu \mathrm{M}$ (b,d).

to explain our observations. In the presence of asymmetric Nodal signalling, actin polymerization in the heart tissue is elevated and clockwise rotation of the cardiac disc and leftward displacement of the linear heart tube are observed, resulting in robust dextral looping in almost all embryos. In the absence of Nodal signalling, actin levels are reduced (shown in Fig. 5), and cardiac rotation 
and leftward displacement of the heart tube are perturbed (shown in Figs 1 and 4). In this situation, directional heart looping is maintained in most embryos due to a tissue-intrinsic mechanism that requires actin polymerization and myosin II activity (shown in Figs 1 and 3-5). When actomyosin activity is blocked, even in the presence of asymmetric Nodal signalling, directional heart looping is prevented (shown in Fig. 5).

Our study provides strong evidence that contrary to current models although Nodal signalling is instructive in promoting correct laterality in the heart, it is not the sole pathway regulating chirality of heart looping. Asymmetric Nodal expression is not only widely conserved between higher vertebrates, but has also been observed in the lower chordates, such as ascidians and lancelets ${ }^{39,40}$. Interestingly, although these ancestral organisms exhibit asymmetric embryonic Nodal expression, accompanied by brain and gut asymmetries, no heart asymmetries are observed in the developing embryo ${ }^{41,42}$. This suggests that ancestrally the heart did not respond to asymmetric Nodal signalling ${ }^{43}$ and it is interesting to speculate that heart asymmetry developed independent of asymmetric Nodal. Supporting this, we demonstrate that chiral looping is intrinsic to the heart tissue itself, even in the absence of Nodal signalling. It is tempting to speculate that regional polarization of cardiomyoctes combined with specific coordinated movement of regionalized cells results in organ-intrinsic heart looping. Corroborating such a mechanism are recent studies demonstrating that various cell lines exhibit intrinsic left-right polarity ${ }^{33,34,44}$, whereas elegant imaging analyses have demonstrated regionalized cell polarity coordination in the mouse heart during development ${ }^{45}$.

Our study demonstrates that subsequent to gastrulation, Nodal signalling is dispensable for directional heart looping. The Nodalrelated genes $s q t$ and $c y c$ are expressed early during gastrulation and are required for mesoderm formation-loss of these factors early during development results in an absence of cardiac mesoderm. Therefore, we cannot exclude a very early role for Nodal during mesoderm formation in promoting left-right asymmetries in the heart; however, to date, no left-right asymmetry in Nodal activity during gastrulation stages has been reported.

Furthermore, we demonstrate that non-muscle myosin II activity and actin polymerization are essential for cardiac looping. Although asymmetric actin bundles in the right side of the chick heart have been shown to be important for regulating dextral looping ${ }^{35}$, the role of non-muscle myosins in total organ laterality and more specifically in heart laterality is beginning to be uncovered. The unconventional non-muscle myosin MYO31DF is required for correct organ laterality in Drosophila ${ }^{29,30}$. Similarly, recent studies in C. elegans and zebrafish suggest that non-muscle myosins are required for symmetry breaking in the early embryo ${ }^{32,46}$. Moreover, asymmetric localization of nonmuscle myosin II was observed in the cardiac fields before linear heart tube formation in zebrafish and mouse embryos ${ }^{36,47}$, and mouse embryos lacking non-muscle myosin II-B exhibit structural cardiac defects that could be related to incomplete heart looping ${ }^{48}$. It is interesting to hypothesize that actomyosinbased cytoskeletal processes may regulate cardiac laterality at a cellular or regional level through asymmetric epithelial morphogenesis. It has been demonstrated that cell-intrinsic laterality is dependent on cytoskeletal components, and that disruption of these components results in loss of directionally biased cellular migration ${ }^{33}$. Furthermore, it has been suggested that polarized actomyosin contractility drives the junctional remodelling required for epithelial morphogenesis ${ }^{49}$. Possibly asymmetric actomyosin contractility in the zebrafish heart drives directional cardiomyocyte migration, resulting in the epithelial remodelling characteristic of heart looping. The mechanism by which such cells acquire polarized actomyosin activity remains unclear, although it is also possible that tissue tension within the heart, driven by addition of cells from the second heart field to the growing heart tube, orients actin localization or myosin recruitment similar to the orientation of tissue polarity by cellular flow in Drosophila axis and wing elongation ${ }^{50,51}$.

Together, these data support that organ laterality is promoted by cytoskeletal remodelling processes that are conserved between species.

\section{Methods}

Fish lines and husbandry. The following zebrafish lines were used: $\mathrm{Tg}(\mathrm{cmlc2:GFP})^{52}$, sfw(Tg:cmlc2:GFP), TL, dnaaf ${ }^{h u 255 H}$ (ref. 18) and hsp70:gal4 (ref. 53). Fish were maintained according to standard laboratory conditions. Animal experiments were approved by the Animal Experimentation Committee (DEC) of the Royal Netherlands Academy of Arts and Sciences. The N-ethyl-Nnitrosourea-mediated mutagenesis screen was performed by mutagenizing $\sim 100$ adult male zebrafish by three to five consecutive treatments with $3 \mathrm{mM} \mathrm{N}$-ethyl-Nnitrosourea. The mutagenized males were crossed to wild-type females to generate F1 families. F1 progeny were subsequently outcrossed to wild-type embryos to create $\sim 300$ F2 families. The F2 families were incrossed and the F3 progeny screened for cardiac laterality defects at 28-32 hpf.

Positional cloning of the sfw mutant. The spaw ${ }^{t 30973}$ allele was mapped by standard meiotic mapping using SSLPs. Sequences for the primers used for SSLP mapping can be found in Supplementary Table S3. The spaw ${ }^{t 30973}$ mutant can be identified using PCR amplification, followed by EcoRI restriction of the PCR product using the following primers: forward primer: $5^{\prime}$-GGACATGATCGT GGAGGAAT- $3^{\prime}$ and reverse primer: $5^{\prime}$-TCAGGAGTCGCCACAGTAGA- $3^{\prime}$; the primers introduce an EcoRI restriction site into the mutant strand.

In situ hybridization. Embryos were fixed overnight in $4 \%$ paraformaldehyde (PFA) and stored in $\mathrm{MeOH}$. Embryos were rehydrated to PBST (PBS plus $0.1 \%$ Tween) and treated with $1 \mu \mathrm{g} \mathrm{ml}^{-1}$ Proteinase $\mathrm{K}$ for between 1 and $20 \mathrm{~min}$ depending on the stage. Embryos were rinsed in PBST, post fixed in $4 \%$ PFA for $20 \mathrm{~min}$, washed $3 \times 5 \mathrm{~min}$ in PBST and pre-hybridized for at least $1 \mathrm{~h}$ in Hybbuffer. Riboprobes were diluted in Hyb-buffer supplemented with transfer RNA and heparin, and incubated with embryos overnight at $70^{\circ} \mathrm{C}$. Following probe removal, embryos were washed stepwise from $\mathrm{Hyb}$ - to $2 \times \mathrm{SSC}$, and subsequently from $0.2 \times$ SSC to PBST. Embryos were blocked for at least $1 \mathrm{~h}$ in PBST supplemented with sheep serum and BSA before being incubated overnight at $4{ }^{\circ} \mathrm{C}$ with anti-digoxygenin antibody. Following antibody removal, embryos were washed extensively in PBST before being washed several times in TBST. Nitro-blue tetrazolium/5-bromo-4-chloro-3-inodyl phosphate was diluted in TBST, added to embryos and protected from the light. Once staining had developed, embryos were washed several times in PBST and fixed overnight in 4\% PFA. Embryos were cleared in $\mathrm{MeOH}$ and mounted in benzylbenzoate:benzylalcohol (2:1) before imaging. Riboprobes were transcribed from linearized template in the presence of 11-UTP. The acta1b in situ was quantified by measuring pixel intensity in the lef side and right side of the cardiac disc and was normalized to background levels (using ImageJ software). Total pixel intensity was then determined and the percentage contribution of left or right side to total intensity was calculated.

mRNA and DNA microinjections. Full-length spaw complementary DNA was cloned into the PCS2 + vector (Addgene). The sfw mutation was introduced into the spaw PCS2 + construct by site-directed mutagenesis using a Quickchange kit (Stratagene). Injection mRNA was transcribed using the SP6 mMessage mMachine kit (Ambion).

The spaw morpholino targeting the intron 2-exon 3 splice site $\left(5^{\prime}\right.$-TGGTAGA GCTTCAACAGACTCTGCA-3') was injected at the one-cell stage at $5 \mathrm{ng}$ (ref. 11). Full-length lefty 1 open reading frame was amplified from wild-type cDNA. The full-length fragment was cloned into a vector containing $14 \times$ UAS promoter and a polyA tail. Embryos were injected at the one-cell stage with $5 \mathrm{pg}$ of UAS:lft 1 and 5 pg of UAS:GFP. Heat-shock experiments were performed by placing embryos in preheated $\mathrm{E} 3$ medium at $37^{\circ} \mathrm{C}$ for $30 \mathrm{~min}$.

Time-lapse analysis. Embryos were dechorionated and mounted in $0.25 \%$ agar ose. Images were acquired using a Leica SP2 confocal microscope and confocal stacks were acquired every $4 \mathrm{~min}$ for $4 \mathrm{~h}$. Embryos were subsequently excised from the agarose and raised to $50 \mathrm{hpf}$. Cells were manually tracked using ImageJ software. Rotation was calculated as an average of angles or rotation from both left and right sides of the heart. A vertical line is drawn between two cells in one side of the heart. A line is drawn between the same cells at the end of the time lapse and the angle between these two lines is taken as a measure of rotation. For velocity analysis, individual cell velocities at each time point during time lapse were calculated in ImageJ, and average velocities for all cells over time in either the anterior or posterior region of the heart were determined. 
Chemical treatments. Embryos were soaked in $100 \mu \mathrm{M}$ of the Nodal inhibitor SB431542 (Sigma), $25 \mu \mathrm{M}$ Blebbistatin (Sigma) or $5 \mu \mathrm{g} \mathrm{ml}^{-1}$ Nocodazole (Sigma) in a final concentration of $1 \%$ dimethyl sulphoxide in E3 medium. After timed treatments, embryos were washed $4 \times 5 \mathrm{~min}$ in fresh $\mathrm{E} 3$ medium before fixation

Bead implants. Agarose beads (Affigel blue, Biorad) were rinsed twice in PBS and incubated for $1 \mathrm{~h}$ at $37^{\circ} \mathrm{C}$ with $50 \mu \mathrm{g} \mathrm{ml}^{-1}$ recombinant mouse Nodal protein (R\&D Systems). Embryos at the 15-18 somite stage were mounted in $0.25 \%$ agarose and beads were inserted under the left eye using a tungsten needle. Embryos were excised from the agarose and fixed at $28 \mathrm{hpf}$.

Heart explants and drug treatments. Hearts were manually dissected from $26 \mathrm{hpf}$ embryos using forceps and placed into supplemented L15 culture medium ( $15 \%$ fetal bovine serum, $0.8 \mathrm{mM} \mathrm{CaCl}_{2}, 50 \mu \mathrm{g} \mathrm{ml}^{-1}$ penicillin, $0.05 \mathrm{mg} \mathrm{ml}^{-1}$ streptomycin, $0.05 \mathrm{mg} \mathrm{ml}^{-1}$ gentomycin $)^{54}$. Explants were incubated at $28.5^{\circ} \mathrm{C}$ for $24 \mathrm{~h}$ and fixed in $4 \%$ PFA overnight. Explants were treated with Blebbistatin $\left(25 \mu \mathrm{M}\right.$, Sigma), Cytochalasin B $\left(5 \mu \mathrm{g} \mathrm{ml}^{-1}\right.$, Sigma) or Nocodazole $\left(100 \mathrm{ng} \mathrm{ml}^{-1}\right.$, Sigma). Explanted hearts were mounted in Vectashield or Mowiol, and imaged using a Leica SPE confocal microscope. Three-dimensional reconstructions were generated using Volocity (Improvision).

Quantitative RT-PCR. Total RNA was extracted from 19.5 hpf wild-type and $s f w$ mutant embryos. cDNA was generated using the RTIII First Strand kit (Invitrogen). Quantitative PCRs were performed using SYBR Green (BioRad) in a BioRad MyIQ PCR machine. Gene expression levels were normalized to gapdh and efla levels. Three biological repeats were used for each genotype and reactions were performed in triplicate.

\section{References}

1. Grande, C. \& Patel, N. H. Nodal signalling is involved in left-right asymmetry in snails. Nature 457, 1007-1011 (2009).

2. Duboc, V. \& Lepage, T. A conserved role for the nodal signaling pathway in the establishment of dorso-ventral and left-right axes in deuterostomes. J. Exper. Zool. B Mol. Dev. Evol. 310, 41-53 (2008).

3. Lohr, J. L., Danos, M. C. \& Yost, H. J. Left-right asymmetry of a nodal-related gene is regulated by dorsoanterior midline structures during Xenopus development. Development 124, 1465-1472 (1997).

4. Levin, M., Johnson, R. L., Stern, C. D., Kuehn, M. \& Tabin, C. A molecular pathway determining left-right asymmetry in chick embryogenesis. Cell 82, 803-814 (1995).

5. Lowe, L. A. et al. Conserved left-right asymmetry of nodal expression and alterations in murine situs inversus. Nature 381, 158-161 (1996).

6. Brennan, J., Norris, D. P. \& Robertson, E. J. Nodal activity in the node governs left-right asymmetry. Genes Dev. 16, 2339-2344 (2002).

7. Levin, M. et al. Left/right patterning signals and the independent regulation of different aspects of situs in the chick embryo. Dev. Biol. 189, 57-67 (1997).

8. Toyoizumi, R., Ogasawara, T., Takeuchi, S. \& Mogi, K. Xenopus nodal related-1 is indispensable only for left-right axis determination. Int. J. Dev. Biol. 49, 923-938 (2005).

9. Conlon, F. L. et al. A primary requirement for nodal in the formation and maintenance of the primitive streak in the mouse. Development 120, 1919-1928 (1994).

10. Rebagliati, M. R., Toyama, R., Fricke, C., Haffter, P. \& Dawid, I. B. Zebrafish nodal-related genes are implicated in axial patterning and establishing left-right asymmetry. Dev. Biol. 199, 261-272 (1998).

11. Long, S., Ahmad, N. \& Rebagliati, M. The zebrafish nodal-related gene southpaw is required for visceral and diencephalic left-right asymmetry. Development 130, 2303-2316 (2003).

12. Smith, K. A. et al. Bmp and Nodal independently regulate lefty1 expression to maintain unilateral Nodal activity during left-right axis specification in zebrafish. PLoS Genet. 7, e1002289 (2011).

13. Gamse, J. T., Thisse, C., Thisse, B. \& Halpern, M. E. The parapineal mediates left-right asymmetry in the zebrafish diencephalon. Development 130, 1059-1068 (2003)

14. Gritsman, K. et al. The EGF-CFC protein one-eyed pinhead is essential for nodal signaling. Cell 97, 121-132 (1999).

15. Mullins, M. C. et al. Genes establishing dorsoventral pattern formation in the zebrafish embryo: the ventral specifying genes. Development 123, 81-93 (1996).

16. Inman, G. J. et al. SB-431542 is a potent and specific inhibitor of transforming growth factor-beta superfamily type I activin receptor-like kinase (ALK) receptors ALK4, ALK5, and ALK7. Mol. Pharmacol. 62, 65-74 (2002).

17. Essner, J. J., Amack, J. D., Nyholm, M. K., Harris, E. B. \& Yost, H. J. Kupffer's vesicle is a ciliated organ of asymmetry in the zebrafish embryo that initiates left-right development of the brain, heart and gut. Development 132, 1247-1260 (2005).

18. van Rooijen, E. et al. LRRC50, a conserved ciliary protein implicated in polycystic kidney disease. J. Am. Soc. Nephrol. 19, 1128-1138 (2008).
19. Baker, K., Holtzman, N. G. \& Burdine, R. D. Direct and indirect roles for Nodal signaling in two axis conversions during asymmetric morphogenesis of the zebrafish heart. Proc. Natl Acad. Sci. USA 105, 13924-13929 (2008).

20. Smith, K. A. et al. Rotation and asymmetric development of the zebrafish heart requires directed migration of cardiac progenitor cells. Dev. Cell 14, 287-297 (2008).

21. Rohr, S., Otten, C. \& Abdelilah-Seyfried, S. Asymmetric involution of the myocardial field drives heart tube formation in zebrafish. Circ. Res. 102, e12-e19 (2008).

22. Manner, J. Cardiac looping in the chick embryo: a morphological review with special reference to terminological and biomechanical aspects of the looping process. Anat. Rec. 259, 248-262 (2000).

23. Voronov, D. A. \& Taber, L. A. Cardiac looping in experimental conditions: effects of extraembryonic forces. Dev. Dyn. 224, 413-421 (2002).

24. Bakkers, J., Verhoeven, M. C. \& Abdelilah-Seyfried, S. Shaping the zebrafish heart: from left-right axis specification to epithelial tissue morphogenesis. Dev. Biol. 330, 213-220 (2009).

25. Campione, M. et al. Pitx2 and cardiac development: a molecular link between left/right signaling and congenital heart disease. Cold Spring Harb. Symp. Quant. Biol. 67, 89-95 (2002).

26. Lenhart, K. F., Holtzman, N. G., Williams, J. R. \& Burdine, R. D. Integration of Nodal and BMP signals in the heart requires FoxH1 to create left-right differences in cell migration rates that direct cardiac asymmetry. PLoS Genet. 9, e1003109 (2013)

27. Bacon, R. L. Self-differentiation and induction in the heart of amblystoma. J. Exp. Zool. 98, 87-125 (1945).

28. Manning, A. \& McLachlan, J. C. Looping of chick embryo hearts in vitro. J. Anat. 168, 257-263 (1990).

29. Hozumi, S. et al. An unconventional myosin in Drosophila reverses the default handedness in visceral organs. Nature 440, 798-802 (2006).

30. Speder, P., Adam, G. \& Noselli, S. Type ID unconventional myosin controls left-right asymmetry in Drosophila. Nature 440, 803-807 (2006).

31. Taniguchi, K. et al. Chirality in planar cell shape contributes to left-right asymmetric epithelial morphogenesis. Science 333, 339-341 (2011).

32. Pohl, C. \& Bao, Z. Chiral forces organize left-right patterning in C. elegans by uncoupling midline and anteroposterior axis. Dev. Cell 19, 402-412 (2010).

33. Wan, L. Q. et al. Micropatterned mammalian cells exhibit phenotype-specific left-right asymmetry. Proc. Natl Acad. Sci. USA 108, 12295-12300 (2011).

34. Xu, J. et al. Polarity reveals intrinsic cell chirality. Proc. Natl Acad. Sci. USA 104 9296-9300 (2007).

35. Itasaki, N., Nakamura, H., Sumida, H. \& Yasuda, M. Actin bundles on the right side in the caudal part of the heart tube play a role in dextro-looping in the embryonic chick heart. Anat. Embryol. 183, 29-39 (1991).

36. Lu, W. et al. Cellular nonmuscle myosins NMHC-IIA and NMHC-IIB and vertebrate heart looping. Dev. Dyn. 237, 3577-3590 (2008).

37. Hinits, Y. et al. Zebrafish Mef2ca and Mef2cb are essential for both first and second heart field cardiomyocyte differentiation. Dev. Biol. 369, 199-210 (2012).

38. Glenn, N. O. et al. The W-loop of alpha-cardiac actin is critical for heart function and endocardial cushion morphogenesis in zebrafish. Mol. Cell Biol. 32, 3527-3540 (2012).

39. Yu, J. K., Holland, L. Z. \& Holland, N. D. An amphioxus nodal gene (AmphiNodal) with early symmetrical expression in the organizer and mesoderm and later asymmetrical expression associated with left-right axis formation. Evol. Dev. 4, 418-425 (2002).

40. Morokuma, J., Ueno, M., Kawanishi, H., Saiga, H. \& Nishida, H. HrNodal, the ascidian nodal-related gene, is expressed in the left side of the epidermis, and lies upstream of HrPitx. Dev. Genes Evol. 212, 439-446 (2002).

41. Jeffries, R. P. S. The Ancestry of the Vertebrates (British Natural History Museum, 1986)

42. Harrison, F. W. \& Ruppert, E. Microscpoic Anatomy of Invertebrates, Hemichordata, Chaetognatha, and the Invertebrate Chordates. Vol. 15 (WileyLiss, 1997).

43. Palmer, A. R. Symmetry breaking and the evolution of development. Science 306, 828-833 (2004).

44. Chen, T.-H. et al. Left-right symmetry breaking in tissue morphogenesis via cytoskeletal mechanics. Circ. Res. 110, 551-559 (2012).

45. Le Garrec, J. F. et al. Quantitative analysis of polarity in 3D reveals local cell coordination in the embryonic mouse heart. Development 140, 395-404 (2012).

46. Wang, G., Manning, M. L. \& Amack, J. D. Regional cell shape changes control form and function of Kupffer's vesicle in the zebrafish embryo. Dev. Biol. 370, 52-62 (2012).

47. Veerkamp, J. et al. Unilateral dampening of bmp activity by nodal generates cardiac left-right asymmetry. Dev. Cell 24, 660-667 (2013).

48. Ma, X. \& Adelstein, R. S. In vivo studies on nonmuscle myosin II expression and function in heart development. Front. Biosci. 17, 545-555 (2012). 
49. Rauzi, M., Lenne, P. F. \& Lecuit, T. Planar polarized actomyosin contractile flows control epithelial junction remodelling. Nature 468, 1110-1114 (2010).

50. Fernandez-Gonzalez, R., Simoes Sde, M., Roper, J. C., Eaton, S. \& Zallen, J. A. Myosin II dynamics are regulated by tension in intercalating cells. Dev. Cell 17, 736-743 (2009).

51. Aigouy, B. et al. Cell flow reorients the axis of planar polarity in the wing epithelium of Drosophila. Cell 142, 773-786 (2010).

52. Huang, C. -J., Tu, C. -T., Hsiao, C. -D., Hsieh, F. -J. \& Tsai, H. -J. Germ-line transmission of a myocardium-specific GFP transgene reveals critical regulatory elements in the cardiac myosin light chain 2 promoter of zebrafish. Dev. Dyn. 228, 30-40 (2003).

53. Scheer, N., Riedl, I., Warren, J. T., Kuwada, J. Y. \& Campos-Ortega, J. A. A quantitative analysis of the kinetics of Gal4 activator and effector gene expression in the zebrafish. Mech. Dev. 112, 9-14 (2002).

54. Choorapoikayil, S., Overvoorde, J. \& den Hertog, J. Deriving cell lines from zebrafish embryos and tumors. Zebrafish 10, 316-325 (2013).

\section{Acknowledgements}

We thank the ZF-Models screen consortium for facilitating the forward genetic screen and Jacqueline Deschamps for critical reading of the manuscript. This work was supported by the Research Council for Earth and Life Sciences (ALW) with financial aid from the Netherlands Organization for Scientific Research (NWO; grant 864.08.009).

\section{Author contributions}

E.S.N. and J.B. designed the study, discussed the results and wrote the manuscript. E.S.N performed the experiments and analysed the data. M.V. identified the sfw mutant. F.T., S.C. and J.d.H. contributed to the heart explant study. A.K.L. and K.S. contributed the tnnt2a mutant data.

\section{Additional information}

Supplementary Information accompanies this paper at http://www.nature.com/ naturecommunications

Competing financial interests: The authors declare no competing financial interests.

Reprints and permission information is available online at http://npg.nature.com/ reprintsandpermissions/

How to cite this article: Noël, E. S. et al. A Nodal-independent and tissue-intrinsic mechanism controls heart-looping chirality. Nat. Commun. 4:2754 doi: 10.1038/ncomms3754 (2013). 